\title{
Eventos adversos em Unidade de Terapia Intensiva Neonatal
}

\author{
Adverse events in a Neonatal Intensive Care Unit \\ Eventos adversos en Unidad de Cuidados Intensivos Neonatales
}

\section{Claudiane Maria Urbano Ventura', João Guilherme Bezerra Alves", Jucille do Amaral Meneses ${ }^{\text {III }}$}

I Instituto de Medicina Integral Prof. Fernando Figueira, Unidade de Terapia Intensiva Neonatal. Recife-PE, Brasil. "Instituto de Medicina Integral Prof. Fernando Figueira, Pós-Graduação Stricto Sensu. Recife-PE, Brasil.

I'I Instituto de Medicina Integral Prof. Fernando Figueira, Unidade de Terapia Intensiva Neonatal, Residência Médica Neonatal. Recife-PE, Brasil.

\section{Submissão 23-01-2011 Aprovação: 16-03-2012}

\section{RESUMO}

Estudo observacional, prospectivo, para determinar a incidência de eventos adversos (EAs) em Unidade de Terapia Intensiva Neonatal (UTIN). Utilizou-se um “instrumento" específico, adaptado do modelo americano utilizado pela Rede Vermont-Oxford. Dos 218 recém-nascidos, 183 (84\%) apresentaram EAs, correspondendo a 2,6 EA/paciente. Distúrbios da termorregulação $(29 \%)$, distúrbios da glicemia $(17,1 \%)$ e Infecção Relacionada à Assistência à Saúde (IRAS) de origem hospitalar (13,5\%) foram os mais frequentes. Alguns EAs apresentaram associação com peso de nascimento $(p<0,05)$. O percentual de IRAS e a extubação não programada foi diretamente proporcional ao tempo de internamento. A incidência de EAs em UTIN é elevada entre os recém-nascidos de muito baixo peso. A qualidade da assistência torna-se primordial na elaboração das estratégias preventivas.

Descritores: Eventos adversos; Recém-nascido; UTI Neonatal; Assistência de enfermagem.

\section{ABSTRACT}

This was a prospective, observational study conducted in a neonatal intensive care unit to determine the incidence of adverse events. A specific trigger tool instrument was used, based on the one from Vermont-Oxford Network. A total of 218 neonates were followed and AEs were detected in 183 (84\%) of them, with a rate of 2,6 AE/patient. Thermoregulation disorders (29\%), disorders of glycemic control $(17,1 \%)$ and nosocomial infections $(13,5 \%)$ were the most frequent. Some AE were associated with birth weight $(p<0.05)$. The accidental extubations and nosocomial infections were associated with hospital days. The incidence of AEs is high, especially among neonates with very low birth weight. Best practices regarding preventive strategies are necessary to improve quality of health care for these infants.

Key words: Adverse events; Newborn; Neonatal Unit.

\section{RESUMEN}

Estudio observacional, prospectivo para determinar la incidencia de eventos adversos (EAs) en la Unidad Neonatal de Cuidados Intensivos (UNCI). Fue utilizado un instrumento específico adaptado del modelo americano Vermont-Oxford. De los 218 recién nacidos, el $84 \%$ presentaron EA, lo que corresponde a 2,6 EA / paciente. Trastornos de la termorregulación (29\%), trastornos de la glicemia $(17,1 \%)$ e infección relacionada con el cuidado de la salud (IRCS) nosocomiales $(13,5 \%)$ fueron los más frecuentes. Algunos presentaron asociación con el peso al nacer $(p<0,05)$. El porcentaje de IRCS y la extubación no programada fue directamente proporcional a la duración de la estancia hospitalaria. La incidencia de EAs en la UNCI es alta entre los recién nacidos de muy bajo peso al nacer. La calidad de la asistencia es fundamental en el desarrollo de estrategias de prevención.

Palabras clave: Eventos adversos; Recién nacido; UCIN; Asistencia de enfermería. 


\section{INTRODUÇÃO}

Os eventos adversos (EAs) são definidos como a presença de complicações indesejáveis decorrentes dos cuidados prestados ao paciente, não atribuídas à evolução natural da doença ${ }^{(1)}$. Em 1955 foi descrito por Barr como sendo ocorrência inevitável, decorrente dos avanços tecnológicos na saúde ${ }^{(2)}$. Atualmente, os EAs constituem um dos maiores desafios para o aprimoramento da qualidade e segurança na área de saúde ${ }^{(3)}$.

O aumento significativo da frequência de eventos adversos tem provocado discussões em âmbito internacional, pois, acarretam acentuado aumento da morbimortalidade, prolongam o tempo de hospitalização e elevam os custos do tratamento ${ }^{(4-5)}$. Alguns estudos epidemiológicos apontam para uma prevalência de 20 a $46 \%$ de EAs em pacientes hospitalizados ${ }^{(6-8)}$. A incidência de EAs nos países desenvolvidos varia de 2,9 a 16,5 por 100 pacientes admitidos em hospitais, provocando a morte de cerca de 100.000 pessoas nos Estados Unidos, a cada ano ${ }^{(9-11)}$. Essa mortalidade chega a ultrapassar as atribuídas aos pacientes com AIDS, câncer de mama e vítimas de atropelamento ${ }^{(11-12)}$.

Os rápidos avanços na medicina neonatal têm contribuído para uma significativa redução da morbimortalidade dos recém-nascidos de alto risco, principalmente os prematuros. No entanto, os avanços tecnológicos que hoje permitem a sobrevivência desses pacientes, paradoxalmente criaram condições favoráveis à ocorrência de complicações. Estima-se que $15 \%$ de todas as admissões em unidades de terapia intensiva neonatal são seguidas de $\mathrm{EAs}^{(13-14)}$.

Entre os EAs mais frequentes destacam-se as infecções nosocomiais, eventos associados a medicamentos, a cateteres intravasculares e eventos relacionados a assistência respiratória do paciente ${ }^{(15)}$. O tempo de internamento do paciente também é considerando como um fator de risco para a ocorrência de EAs, aumentando em $6 \%$ a cada dia de internamento, principalmente em unidade de terapia intensiva, pois o numero de intervenções é relativamente elevada ${ }^{(16)}$.

Considerando os EAs com fator de impacto para saúde e como parte de uma série de medidas colaborativas para ajudar na mensuração da qualidade e segurança dos pacientes nas UTINs, a Rede Neonatal Norte-Americana Vermont-Oxford realizou uma pesquisa para avaliar a real incidência destes EAs nestas unidades. No ano de 2006, Sharek et al. construíram um "instrumento de gatilho" específico para UTINs, composto por eventos sentinela para serem identificados nos prontuários dos RNs. Neste estudo a taxa observada foi de $0,74 \mathrm{EA} /$ paciente, sendo maior nos recém-nascidos com peso $<1500$ g ou com IG $<28$ semanas $^{(17-18)}$.

Mais recentemente, Lugi e col na França, através de um estudo prospectivo obtiveram uma taxa semelhante ao estudo americano com 0,78 EA/paciente. Os principais fatores de risco associados à presença de EAs foram baixo peso ao nascimento, presença de cateter intravascular e ventilação mecânica assistida ${ }^{(19)}$.

A presença de EAs em UTI neonatais tem se tornado um importante desafio na assistência aos recém-nascidos. No entanto, os estudos epidemiológicos que abordem este tema, ainda são escassos, principalmente pela dificuldade de metodologia empregada. Dessa forma, este estudo teve como objetivo principal determinar a frequência de EAs, aplicando a metodologia do "instrumento de gatilho" Americano, em uma UTIN de um país em desenvolvimento(20).

\section{MÉTODO}

O presente estudo foi realizado na Unidade de Terapia Intensiva Neonatal (UTIN) do Instituto de Medicina Integral Prof. Fernando Figueira (IMIP). A Unidade Neonatal do IMIP dispõe de 50 leitos, dos quais 18 leitos são destinados à UTIN.

Foi realizado um estudo observacional, prospectivo tendo como amostra todos os recém-nascidos admitidos no período de março a agosto de 2009. A amostra necessária para a realização deste estudo foi calculada através do programa STATCALC do software de domínio público EPI-Info versão 3.4.3, da Organização Mundial de Saúde (CDC), prevendo uma incidência de EAs com base na taxa de infecção relacionada à assistência à saúde de origem hospitalar de $17 \%$ para o ano do estudo. Considerando um intervalo de confiança de $95 \%$ e prevendo as possíveis perdas, a amostra final foi composta por 218 recém-nascidos admitidos.

Foram considerados elegíveis os recém-nascidos com tempo de internamento superior a dois dias. Todos os participantes do estudo foram acompanhados diariamente na UTIN por um dos pesquisadores até a saída da Unidade, por transferência, alta ou óbito.

A coleta dos dados foi realizada através da utilização de um instrumento específico do tipo "instrumento de gatilho", composto por eventos sentinela, para a captação e identificação de eventos adversos na UTIN, adaptado do instrumento desenvolvido por Sharek et al. composto por 14 tipos de eventos passiveis de identificação. Dos 14 tipos de eventos adversos selecionados no presente estudo, 12 foram semelhantes ao estudo de Sharek et al.: infecção relacionada à assistência à saúde de origem hospitalar, extubação não programada, pneumotórax, complicações relacionadas ao cateter intravascular, presença de trombo arterial ou venoso, hipotensão arterial, insuficiência renal aguda, convulsões, distúrbios da glicemia, hemorragia peri-intraventricular, enterocolite necrotizante, óbito ${ }^{(21)}$. Optamos por acrescentar mais dois tipos de eventos que não foram contemplados pelo estudo americano, os distúrbios de termorregulação e lesão de septo nasal, totalizando $14 \mathrm{EAs}^{(22)}$.

Durante a coleta diária e na busca da identificação dos EAs, o pesquisador notificava no formulário apenas a primeira ocorrência de cada tipo de evento em cada paciente. Todos os EAs foram classificados de acordo com a sua gravidade, baseada na classificação da Coordenação Nacional Americana para Notificação de Evento Adverso Relacionada a Medicamentos (NCCMERP), que utiliza cinco categorias $(E, F, G, H, I)$, em ordem crescente de gravidade ${ }^{(23)}$.

- Categoria E: quando o evento adverso contribui ou resulta em danos temporários;

- Categoria F: quando o evento adverso contribui ou resulta em danos temporários e requer prolongamento do tempo de hospitalização; 
- Categoria G: Quando o evento adverso contribui ou resulta em danos permanentes;

- Categoria H: Quando o evento adverso contribui ou resulta em danos, que requerem intervenção para manutenção da vida;

- Categoria I: Quando o evento adverso é o próprio óbito ${ }^{(23)}$.

Na análise bivariada, as variáveis categóricas foram comparadas em tabelas de contingência, utilizando-se os testes qui-quadrado de associação, quando pertinente. O método Kaplan Méier foi utilizado para analisar a ocorrência de IRAS de origem hospitalar e extubação não programada associada ao tempo de internamento. Em todas as etapas da análise foi adotado o nível de significância de 0,05.

O estudo recebeu previamente a aprovação do Comitê de Ética em Pesquisa do IMIP, Protocolo n 1127 , e todos os responsáveis legais dos sujeitos da pesquisa assinaram o termo de consentimento livre e esclarecido.

\section{RESULTADOS}

Participaram do estudo 218 recém-nascidos, com um total de 2958 paciente/dias. Nesta população, 183 (84\%) apresentaram algum tipo de evento adverso. Por se tratar de uma população de recém-nascidos de alto risco, 187 (85,8\%) apresentavam idade gestacional $<37$ semanas e 122 (56\%) peso ao nascimento $<$ 1500g. O tempo de internamento na UTIN variou de 2 a 72 dias, com uma média de 13.5 dias/paciente ( $D P=14.3$ ), resultando numa taxa de 195 EAs por 1000 paciente/dias.

Foram identificados 579 eventos adversos resultando numa taxa de 2,6 EA/paciente. Na Tabela 1, foram identificados os tipos de eventos, sendo os distúrbios da termorregulação (29\%), os distúrbios da glicemia $(17,1 \%)$, a infecção relacionada à assistência à saúde de origem hospitalar (13,5\%), seguidos de extubação não programada $(10, \%)$, considerados os mais frequentes.

Em relação aos distúrbios de termorregulação, 65,9\% dos RNs apresentaram hipotermia e 5,4\% hipertermia sendo que, na grande maioria dos casos, a hipertermia era secundária a
Tabela 1- Distribuição percentual dos eventos adversos em recém-nascidos na UTIN do IMIP. Recife-PE, 2008.

\begin{tabular}{l|c}
\hline Tipos de EAs & N (\%) \\
\hline Distúrbios de termorregulação & $167(29,0)$ \\
\hline Distúrbios da glicemia & $99(17,1)$ \\
\hline IRAS de origem hospitalar & $78(13,5)$ \\
\hline Extubação não programada & $58(10,0)$ \\
\hline Óbito & $36(6,2)$ \\
\hline Lesão de septo nasal & $35(6,0)$ \\
\hline Relacionado ao Cateter Venoso Central & $35(6,0)$ \\
\hline Hipotensão & $24(4,1)$ \\
\hline Insuficiência renal & $23(4,0)$ \\
\hline Convulsões/estado do mal convulsivo & $9(1,6)$ \\
\hline Enterocolite necrotizante & $6(1,0)$ \\
\hline Hemorragia intra-periventricular & $3(0,5)$ \\
\hline Pneumotórax & $3(0,5)$ \\
\hline Trombo & $3(0,5)$ \\
\hline Total & $579(100,0)$ \\
\hline
\end{tabular}

tentativa de correção da hipotermia. O distúrbio da glicemia foi o segundo EA mais frequente, dos quais $34,3 \%$ dos RNs apresentaram a hipoglicemia. A Infecção relacionada à assistência a saúde (IRAS) de origem hospitalar esteve presente em 78 recém-nascidos internados, correspondendo a 13,5\% dos EAs. A extubação não programada foi o quarto evento adverso mais frequente neste estudo (10\%), e o maior percentual dessas extubações ocorreu de forma acidental (62\%). A obstrução mecânica por rolha foi responsável pelo restante dos casos(38\%).

Na Tabela 2, observa-se uma associação entre alguns tipos EAs e o peso de nascimento, sendo este achado significativo $(p<0,05)$ para IRAS de origem hospitalar, extubação não-programada, hipotensão, distúrbios da glicemia, distúrbio de termorregulação, lesões de septo nasal e óbito. Todos esses eventos apresentaram uma ocorrência inversamente

Tabela 2- Associação dos eventos adversos com o peso ao nascimento dos recém-nascidos na UTIN do IMIP. Recife-PE, 2008.

\begin{tabular}{|c|c|c|c|c|c|}
\hline \multirow[b]{2}{*}{ Eventos Adversos } & \multicolumn{5}{|c|}{ Peso ao nascimento (\%) } \\
\hline & $\begin{array}{c}<1000 \mathrm{~g} \\
\mathrm{n}(\%)\end{array}$ & $\begin{array}{c}1001-1500 \mathrm{~g} \\
\mathrm{n}(\%)\end{array}$ & $\begin{array}{c}\text { 1501- } 2500 \mathrm{~g} \\
\mathrm{n}(\%)\end{array}$ & $>\underset{\mathrm{n}(\%)}{2500 \mathrm{~g}}$ & $p^{*}$ \\
\hline IRAS de origem hospitalar & $33(42,3)$ & $25(32,1)$ & $15(19,2)$ & $5(6,4)$ & $<0,000$ \\
\hline Extubação não programada & $21(36,2)$ & $21(36,2)$ & $10(17,3)$ & $6(10,3)$ & 0,003 \\
\hline Relacionados ao Cateter Venoso Central & $13(37,1)$ & $13(37,1)$ & $6(17,1)$ & $3(8,7)$ & 0,078 \\
\hline Insuficiência renal & $9(36)$ & $9(36)$ & $4(16)$ & $3(12)$ & 0,096 \\
\hline Hipotensão & $12(50)$ & $7(29,2)$ & $4(16,6)$ & $1(4,2)$ & 0,002 \\
\hline Distúrbio da glicemia & $35(35,3)$ & $36(36,4)$ & $17(17,2)$ & $11(11,1)$ & $<0,001$ \\
\hline Distúrbios de termorregulação & $51(30,5)$ & $56(33,5)$ & $40(24)$ & $20(12)$ & $<0,001$ \\
\hline Lesão de septo nasal & $17(48,6)$ & $13(37,1)$ & $5(14,3)$ & - & $<0,001$ \\
\hline Óbito & $15(42)$ & $9(25)$ & $5(14)$ & $7(19)$ & 0,002 \\
\hline
\end{tabular}

*Teste de qui-quadrado de tendência 
Figura 1. Sobrevida de recém-nascidos livre da ocorrência de infecção relacionada à assistência à saúde de origem hospitalar na UTIN do IMIP. Método de Kaplan-Meier com IC 95\%. Recife-PE, 2008.

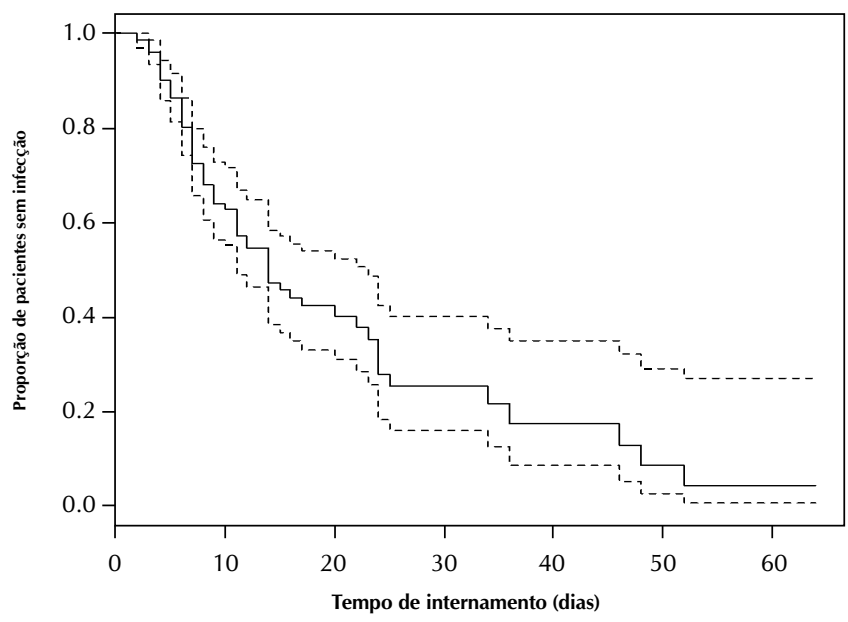

Figura 2 - Sobrevida de recém-nascidos livre da extubação não programada na UTIN do IMIP. Método de Kaplan-Meier com IC 95\%. Recife-PE, 2008.

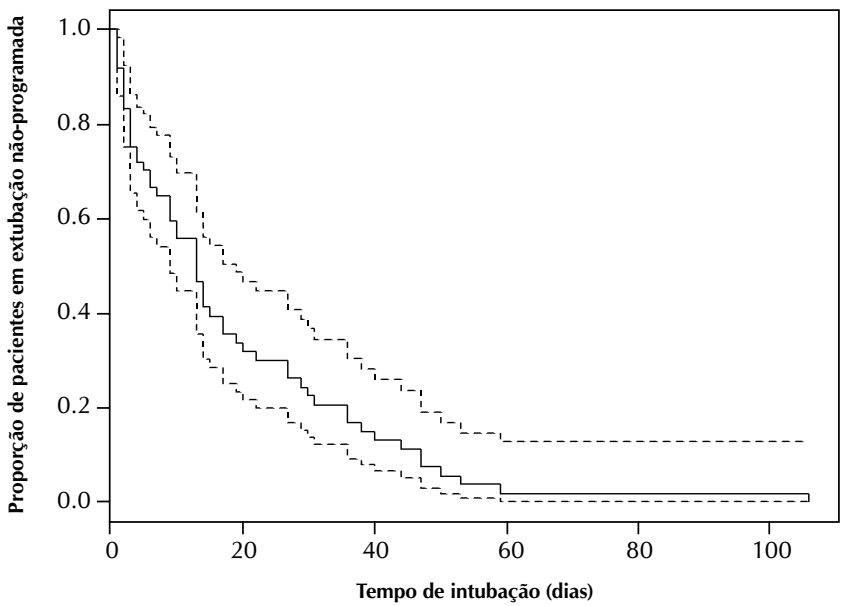

proporcional ao peso de nascimento. Apenas insuficiência renal e os eventos relacionado ao cateter venoso central não teve associação significativa.

A avaliação da sobrevida de recém-nascidos livre da ocorrência de eventos adversos através do método Kaplan Meier, foi demonstrada na Figura 1, onde se observa que, à medida que se prolonga o tempo de internamento hospitalar, diminui o percentual de recém-nascidos livres da ocorrência de IRAS de origem hospitalar, sendo esta redução mais significativa nos primeiros 20 dias de internamento.

Sabendo-se que o tempo prolongado de recém-nascidos submetidos a ventilação mecânica invasiva por intermédio de um tubo orotraqueal, aumenta o risco para ocorrência da extubação não programada. Neste estudo, quanto maior o tempo de entubação traqueal, maior foi o risco de ocorrência
Figura 3 - Distribuição percentual das categorias dos eventos adversos de acordo com a sua gravidade na UTIN do IMIP. Recife-PE, 2008.

\section{Categoria dos eventos adversos de acordo com sua gravidade}

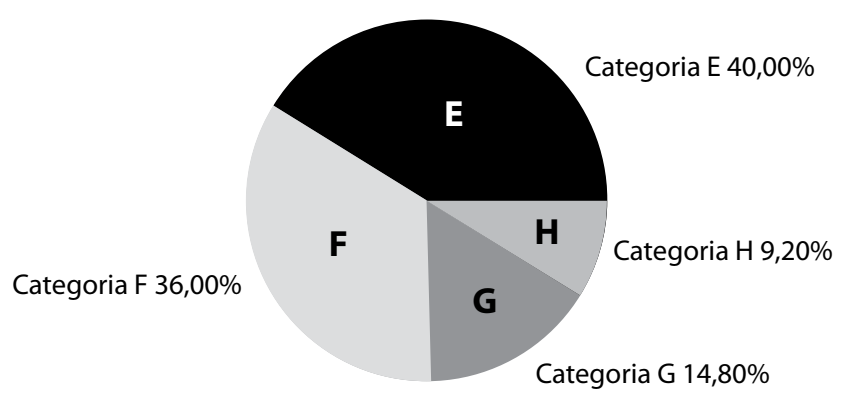

da extubação não programada. A Figura 2 demonstra que, no vigésimo dia de entubação, aproximadamente $35 \%$ dos recém-nascidos estiveram livres do risco deste tipo de evento adverso.

Os EAs foram classificados de acordo com sua gravidade, sendo $76 \%$ classificados nas categorias $E$ e $F$, resultando em danos temporários e/ou prolongamento do tempo de internamento hospitalar, nenhum dos eventos adversos identificados através do instrumento de gatilho, foi classificado na categoria I, resultando em óbito (Figura 3)

\section{DISCUSSÃO}

Neste estudo, foi utilizado um "instrumento de gatilho" para identificação e notificação de alguns tipos específicos de EAs. O único estudo encontrado na literatura com EA em UTIN empregando a metodologia do "instrumento de gatilho" foi o de Sharek et al que, revisando prontuários em 15 UTIN americanas, encontraram uma taxa de 0,74 EA por paciente ${ }^{(17-18)}$. A taxa de EAs observada neste estudo foi de 2,6/ paciente, sendo 3,5 vezes maior do que a encontrada no estudo americano.

Os estudos relacionados à frequência de EAs demonstram que a identificação desses eventos varia de acordo com a metodologia empregada ${ }^{(24)}$. Neste estudo o emprego da metodologia prospectiva para identificação dos EAs, pode ter contribuído para a maior taxa de EAs, que a referida no estudo americano "Vermont-Oxford", o qual utilizou uma análise retrospectiva ${ }^{(17)}$. Ao mesmo tempo, existem dois estudos observacionais prospectivos, nos quais a taxa de EAs em UTINs encontrada variou de 0,4 e 0,78/paciente, sendo que, neste estudo, não foi utilizado o "instrumento de gatilho" para identificação dos EAs e a equipe médica estava ciente do estu$\mathrm{do}^{(18)}$. Acreditamos que a maior frequência de EAs observados em nosso estudo também está relacionada com alguns tipos de eventos selecionados, os quais não estavam contemplados no estudo de Sharek et al, como os distúrbios de termorregulação e lesão de septo. 
Os distúrbios de termorregulação ainda constituem um fator crítico na assistência ao recém-nascido, mesmo em países desenvolvidos. Laptook e col., ao estudar 5.277 pré-termos de muito baixo peso nascidos em 15 centros norte-americanos em 2002-2003, encontraram que 36\% desses pacientes apresentaram temperatura $<35,9^{\circ} \mathrm{C}$ e $11 \%$ igual ou superior a $37^{\circ} \mathrm{C}$. Em uma maternidade no Texas entre 2003 e 2007, constatou-se hipotermia em $54 \%$ dos 2.183 prematuros abaixo de 34 semanas $^{(25-26)}$.

Estudo realizado com recém-nascidos externos admitidos na emergência pediátrica de um hospital de referencia demonstrou que a hipotermia foi um fator de risco significativo para mortalidade intra-hospitalar com um $R R$ 3,09 no grupo exposto à hipotermia ${ }^{(27)}$.

Considerando que os distúrbios de termorregulação, principalmente a hipotermia, contribuíram para elevação do percentual de EAs neste estudo, a não inclusão deste tipo de evento reduziria a taxa de 2,6 para 1,8 EAs/paciente, equivalente a uma redução total de $30 \%$. Portanto, diante da magnitude deste evento na população neonatal, faz-se necessário estabelecer melhores praticas e intervenções para a redução desta ocorrência.

Apesar de o método utilizado ser eficaz na identificação de EAs, ele deve ser específico para populações diferentes e determinadas clínicas (UTI Neonatal e UTI Pediátrica). O uso de um "instrumento de gatilho" desenvolvido para população adulta, quando aplicado em uma população pediátrica, tende a subestimar o percentual de EAs, em pelo menos $20 \%$ dos $\operatorname{casos}^{(18)}$.

Os recém-nascidos são mais vulneráveis a apresentar desequilíbrio da glicemia (hipoglicemia e hiperglicemia) nos primeiros dias de vida. Este foi o segundo evento adverso mais frequente $(17,1 \%)$ na população estudada. No estudo de Sharek et al, apenas a hiperglicemia foi avaliada, estando presente em $1,6 \%$ dos casos. Se considerarmos apenas a hiperglicemia, haverá uma redução no percentual de $17,1 \%$ para $11 \%$, mesmo assim, este percentual continua mais elevado que o estudo anterior ${ }^{(17)}$. Sabendo que a hiperglicemia é comum em RNMBP, observamos que, no estudo americano, $18 \%$ da população eram de RNMBP enquanto que na nossa amostra este percentual foi de $55 \%$, o que pode ter contribuído para um maior número deste tipo de evento.

IRAS de origem hospitalar foi considerado o EA mais frequente $(27,8 \%)$ no estudo norte americano. Ligi e col relataram que as IRAS foi o segundo evento mais frequente $(23,2 \%)$ e no estudo de Kugelman e col este evento foi o terceiro mais frequente $(15 \%)$, denotando a importância da identificação deste tipo de evento adverso em UTIN, pois, neste estudo, este evento foi o mais significativo, presente em 13,5\% dos casos, apresentando uma associação inversamente proporcional com o peso de nascimento principalmente nos RNMBP.

Estima-se que, no Brasil, $60 \%$ da mortalidade infantil ocorra no período neonatal, sendo a sepse uma das principais causas. Alguns estudos regionais mostraram índices médios de 25/1000pacientes/dia(28).

Adicionalmente, sua ocorrência esteve relacionada com o tempo de internamento hospitalar, onde cabe ressaltar que o risco de infecção é diretamente proporcional à gravidade da doença, as condições nutricionais, a natureza dos procedimentos diagnósticos ou terapêuticos, bem como ao tempo de internação, dentre outros aspectos ${ }^{(29-30)}$.

Nos estudos que avaliam a qualidade na assistência à saúde, a taxa de extubação não programada é um dos indicadores de processo mais importante, pois neste estudo, observou-se que a extubação não programada, de forma acidental ou mecânica por obstrução (rolha), contribuiu com 10,0\% dos EAs estando associada ao peso de nascimento, principalmente nos recém-nascidos menores que $1500 \mathrm{~g}$ (Tabela 2), já no estudo de Sharek e col a extubação não programada contribuiu com 8,3\% dos EAs, taxa considerada elevada para UTINs de referência ${ }^{(17)}$.

Considerando que a extubação não programada são passíveis de prevenção. A sua ocorrência poderá resultar no prolongamento do tempo de ventilação mecânica, risco de infecção, diminuição do sucesso da extubação traqueal e aumento da ocorrência de broncodisplasia pulmonar ${ }^{(31)}$.

O tempo de internação do paciente também é um fator de risco para eventos adversos, o que aumenta em $6 \%$ a cada dia de internação, principalmente em unidades de terapia intensiva, nas quais o número de procedimentos e manipulações é relativamente maior ${ }^{(16)}$.

A ocorrência de EAs está intrinsecamente relacionada com a qualidade da assistência e a segurança dos pacientes. Sabe-se que a ocorrência de EAs prolonga o tempo de internamento hospitalar e pode resultar em danos ou sequelas temporárias e permanentes. Além disso, os eventos considerados mais graves necessitam muitas vezes de uma intervenção para a manutenção da vida ou podem até mesmo resultar em morte ${ }^{(14)}$.

Em alguns estudos, preconiza-se a classificação da Coordenação Nacional Americana para Notificação de Evento Adverso Relacionada a Medicamentos (NCCMERP) para classificar estes eventos quanto a sua gravidade ${ }^{(19)}$. Neste estudo, os eventos adversos foram responsáveis em $76 \%$ dos casos, por danos temporários e/ou prolongamento do tempo de internamento hospitalar, classificados nas categorias E e F, sendo estes achados semelhantes no estudo realizado pela Vermont Oxford com $77,3 \%$.

A maioria dos autores refere que o maior percentual dos eventos adversos ocorridos são preveníveis. No entanto, as pesquisas em UTINs demonstram índices que variam de $34 \%$ a $90 \%$. No nosso estudo, $87 \%$ dos EAs foram considerados previnível, denotando a importância da necessidade de estabelecer estratégias efetivas para minimizar sua ocorrência ${ }^{(23)}$.

Não podemos deixar de reconhecer algumas limitações do estudo. Acreditamos que a limitação mais importante é, sem dúvida, a inexistência de um padrão ouro na identificação de EAs em UTINs, para comparar como os nossos resultados. No entanto, como o estudo de Sharek et al. foi desenvolvido na Rede Norte Americana Vermont Oxford, reconhecida pela sua excelência na qualidade da assistência neonatal, mesmo reconhecendo as diferenças metodológicas, optamos por comparar os nossos resultados com este estudo ${ }^{(17-18)}$.

Uma segunda limitação, concernente ao "instrumento de gatilho" utilizado neste estudo, é que este instrumento ainda não foi validado no Brasil, pois esta metodologia foi 
desenvolvida nos EUA, que apresentam um quadro de assistência neonatal diverso do nosso, o que leva a uma diferença na incidência e nos tipos de EAs, de acordo com a realidade institucional.

Em terceiro lugar, os EAs observados no estudo podem estar subdimensionados, pois, mesmo com o uso do "instrumento de gatilho", sua identificação ainda é subjetiva e variável, entre os observadores. Somando-se a isso, não podemos descartar a possibilidade do registro incompleto, nos prontuários, da evolução clínica, procedimentos e ocorrências pela equipe de profissionais envolvidos na assistência ao recém-nascido.

\section{CONCLUSÃO}

Concluímos que é elevada a incidência de EAs em uma UTIN de referência, especialmente entre os recém-nascidos de muito baixo peso. A metodologia empregada, através do instrumento de gatilho desenvolvido para recém-nascidos em UTINs, possibilitou a identificação dos EAs mais frequentes nesta população. É importante ressaltar que a maioria dos EAs são passíveis de prevenção, através de intervenções simplificadas e accessíveis à equipe multidisciplinar.

Até o presente momento, não temos conhecimento de outros estudos realizados no Brasil avaliando os EAs em UTIN, utilizando esta metodologia. Este estudo identificou a ocorrência de EAs em uma UTIN de referência no Nordeste do Brasil e os resultados obtidos retratam a real dimensão e magnitude do problema em nível institucional.

Novas pesquisas, com metodologias específicas para a determinação de EAs, como empregada neste estudo, devem ser realizados em outras unidades neonatais, para a comprovação desses resultados e o desenvolvimento de estratégias para a prevenção desse importante problema relacionado com a qualidade da assistência à saúde.

\section{REFERÊNCIAS}

1. Leape LL. Reporting of adverse events. N Engl J Med 2002;347:1633-8.

2. BARR, DP. Hazards of modern diagnosis and therapy-the price we pay. JAMA 1955;159:1452-6.

3. World Health Organization. Patient safety: Daped assessment methods for estimating hazards. Genebra; 2003. E N de Vries,1 M A Ramrattan,2 S M Smorenburg,2 D J Gouma,1 M A Boermeester1Qual The incidence and nature of in-hospital adverse events: a systematic review Qual Saf Health Care 2008;17:216-23.

4. Gallotti, R. M. D. Eventos adversos e óbitos hospitalares em serviço de emergências clínicas de um hospital universitário terciário: um olhar para a qualidade da atenção. São Paulo. Tese [Doutorado] - Faculdade de Medicina da Universidade de São Paulo; 2003.

5. Classen DC, Pestotnik SL, Evans RS, Burke JP. Computerized surveillance of adverse drug events in hospital patients. JAMA 1991;266:2847-51.

6. Steel K, Gertman PM, Crescenzi C, Anderson J. latrogenic illness on a general medical service at a university hospital. N Engl J Med 1981;304:638-42.

7. Andrews LB, Stocking C, Krizek T, Gottlieb L, Krizek C, Vargish T, Siegler,M. An alternative strategy for studying adverse events in medical care. Lancet 1997;349:309-13

8. Schimmel EM. The hazards of hospitalization. Ann Intern Med 1964; 60:100-9

9. Thomas EJ, et al. Incidence and types of adverse events and negligent care in Utah and Colorado. Med Care 2002;38:261-71.

10. Donna W, Eric T, Jane H, Stuart A, Brennan TA. Adverse events and preventable adverse events in children. Pediatr 2005: 115(1)155-60.
11. Kohn LT, Corrigan JM, Donaldson MS (eds). To err is human: building a safer health system. Committee on Quality of Health Care in America, Institute of Medicine. Washington: National Academy Press; 2000.

12. Kraman S.S, et al. Risk management: extreme honesty may be the Best policy. Ann Intern Med 1999;131:963-7.

13. Kohn LT. To err is human: building a safer health care system. Institute of Medicine; 1999.

14. Carvalho. M, Vieira. A. A. Erro médico em pacientes hospitalizados. J Pediatr 2002;78(4):261-8

15. Tibby SM, Correa-West J, Durward A, Ferguson L, Murdoch IA. Adverse events in a paediatric intensive care unit: relationship to workload, skill mix and staff supervision. Intensive Care Med 2004; 30:1160-6.

16. Pietro D. A., Shyavitz L. J., Smith R. A., et al. Detecting andreporting medical errors: why the dilemma. $\mathrm{Br} J$ Med 2000;320: 794-6.

17. Weingart. S. N, Wilson R. M, Gibberd R.W, Harrison B. Epidemiology of medical error. Br J Med 2000;320:774-7.

18. Sharek PJ, Classen D. The incidence of adverse events and medical error in pediatrics. Pediatr Clin North Am 2006;53(6):1067-77.

19. Sharek PJ, Horbar JD, Mason W, Bisarya H, Thurm CW, Suresh G, Gray JE, Edwards WH, Goldmann D, Classen D. Adverse events in the neonatal intensive care unit: development, testing, and findings of an NICU-focused trigger tool to identify harm in North American NICUs. Pediatr 2006;118(4):1332-40.

20. Vries EN, Ramrattan MA, Smorenburg SM, Gouma DJ, Boermeester DJ. The incidence and nature of hospital in-hospital adverse events: a systematic review. Qual. Saf. Health Care 2008;17:216-23. 
21. Resar RK, Rozich JD, Classen DC. Methodology and rationale for the measurement of harm with trigger tools. Qual Saf Health Care 2003;12:39-45.

22. Institute for healthcare improvente. Trigger tool for mesasuring adverse events. Aalable at. www.ihi.org.accessed. may. 2008

23. Rozich JD, Haraden CR, Resar RK. Adverse drug event trigger tool: a practical methodology for measuring medication related harm. Qual Saf Health Care 2003;12:194-200.

24. National Coordinating Council for Medication Error Repoting and Prevention [homepage na internet] Taxonomy of medication errors. [acesso em 10 maio 2006] Disponível em: www.nccmerp.orgaccessed.

25. Resar RK, Rozich JD, Simmonds T, Haraden CR. A trigger tool to identify adverse events in the intensive care unit. Jt Comm J Qual Patient Saf 2006;32(10):585-90.

26. Hoehn T, Hansmann G, Bührer C, Simbruner G, Gunn AJ, Yager J, Levene M, Hamrick SE, Shankaran S, Thoresen M.
Therapeutic hypothermia in neonates. Review of current clinical data, ILCOR recommendations and suggestions for implementation in neonatal intensive care units. Resusc $2008 ; 78(1): 7-12$.

27. Abbot R. Laptook, MD. The Neoneuronetwork Hypothermia Randomized Controlled Trial. Pediatr 2010;126(4):e965-6

28. Silveira. S M M.; Melo. M J G.; Vidal S. A; et al. Hipotermia na damissão: fator de riaco para mortalidade em recém-nascidos transferidos para o IMIP. 2001. p. 130

29. Ferraz S V C. Incidência de sepse neonatal segundo diferentes critérios diagnósticos no instituto materno infantil de Pernambuco, 1999. Pediatr Atual 2002;15:14-8.

30. Couto R. C; Pedrosa T.M.G. Guia pratico de infecção hospitalar. 2 ed. Belo Horizonte: MEDSI; 2004. 356p.

31. Vicent JL - Nosocomial infections in adult intensive-care units. Lancet 2003;362:2068-2077.

32. Antunes $\mathrm{L} C \mathrm{O}$ et al. Falha na extubação de prematuros em UTI neonatal. Salusvita 2003;22(3): 3833-93. 